\title{
Duration of antibiotic therapy for critically ill patients with bloodstream infections: A retrospective cohort study
}

\author{
Thomas C Havey $\mathrm{MD}^{1}$, Robert A Fowler $\mathrm{MDCM} \mathrm{MS}^{1,2,3}$, Ruxandra Pinto $\mathrm{PhD}^{3}$, \\ Marion Elligsen $\mathrm{BPh}^{4}$, Nick Daneman $\mathrm{MD} \mathrm{MSc}^{1,3,5}$
}

TC Havey, RA Fowler, R Pinto, M Elligsen, N Daneman. Duration of antibiotic therapy for critically ill patients with bloodstream infections: A retrospective cohort study. Can J Infect Dis Med Microbiol 2013;24(3):129-137.

BACKGROUND: The optimal duration of antibiotic treatment for bloodstream infections is unknown and understudied.

METHODS: A retrospective cohort study of critically ill patients with bloodstream infections diagnosed in a tertiary care hospital between March 1, 2010 and March 31, 2011 was undertaken. The impact of patient, pathogen and infectious syndrome characteristics on selection of shorter ( $\leq 10$ days) or longer ( $>10$ days) treatment duration, and on the number of antibiotic-free days, was examined. The time profile of clinical response was evaluated over the first 14 days of treatment. Relapse, secondary infection and mortality rates were compared between those receiving shorter or longer treatment.

RESULTS: Among 100 critically ill patients with bloodstream infection, the median duration of antibiotic treatment was 11 days, but was highly variable (interquartile range 4.5 to 17 days). Predictors of longer treatment (fewer antibiotic-free days) included foci with established requirements for prolonged treatment, underlying respiratory tract focus, and infection with Staphylococcus aureus or Pseudomonas species. Predictors of shorter treatment (more antibiotic-free days) included vascular catheter source and bacteremia with coagulasenegative staphylococci. Temperature improvements plateaued after the first week; white blood cell counts, multiple organ dysfunction scores and vasopressor dependence continued to decline into the second week. Among 72 patients who survived to 10 days, clinical outcomes were similar between those receiving shorter and longer treatment.

CONCLUSION: Antibiotic treatment durations for patients with bloodstream infection are highly variable and often prolonged. A randomized trial is needed to determine the duration of treatment that will maximize cure while minimizing adverse consequences of antibiotics.

Key Words: Antibacterials; Antibiotic stewardship; Bacteremia; Bacterial infections; Critical care

\section{La durée de l'antibiothérapie de patients gravement malades atteints d'une bactériémie : une étude de cohorte rétrospective}

HISTORIQUE : On ne connaît pas la durée optimale de
l'antibiothérapie des bactériémies, qui est trop peu étudiée.
MÉTHODOLOGIE : Les chercheurs ont entrepris une étude de
cohorte rétrospective de patients gravement malades atteints d'une
bactériémie qui ont été diagnostiqués dans un hôpital de soins ter-
tiaires entre le $1^{\mathrm{er}}$ mars 2010 et le 31 mars 2011 . Ils ont examiné les
conséquences des caractéristiques du patient, du pathogène et du syn-
drome infectieux sur le choix d'un traitement plus court (dix jours ou
moins) ou plus long (plus de dix jours) et sur le nombre de jours sans
prise d'antibiotiques. Ils ont évalué le profil temporel de la réponse
clinique au cours des 14 premiers jours du traitement et ont comparé le
taux de récidives, d'infections secondaires et de mortalité entre ceux
qui recevaient un traitement plus court et plus long. RÉSULTATS : Chez 100 patients gravement malades ayant une bactériémie, l'antibiothérapie avait une durée médiane de 11 jours, mais était très variable (plage interquartile de 4,5 à 17 jours). Les prédicteurs d'un traitement plus long (moins de journées sans antibiotiques) comprenaient des foyers comportant des exigences établies de traitement prolongé, un accent sur une infection sous-jacente des voies respiratoires et une infection par des espèces de Staphylococcus aureus ou de Pseudomonas. Les prédicteurs d'un traitement plus court (plus de journées sans antibiotiques) incluaient une origine dans un cathéter vasculaire et une bactériémie à staphylocoques négative à la coagulase. L'atténuation de la température a plafonné au bout de la première semaine, tandis que la numération des globules blancs, les indices de dysfonction multiorganique et la dépendance aux vasopresseurs a continué de décliner pendant la deuxième semaine. Chez les 72 patients qui ont survécu jusqu'à dix jours, les issues cliniques étaient similaires chez ceux qui recevaient un traitement plus court ou plus long.

CONCLUSION : La durée de l'antibiothérapie des patients atteints d'une bactériémie est très variable et souvent prolongée. Il faudra procéder à un essai aléatoire pour déterminer la durée du traitement qui favorisera la guérison au maximum tout en limitant le plus possible les conséquences négatives des antibiotiques.

due to high prevailing rates of antibiotic resistance) has been associated with unfavourable outcomes $(8,9)$. However, even with the prompt administration of adequate antimicrobials, mortality due to bloodstream infection in the intensive care setting remains high and the appropriate duration of therapy is poorly defined.

Prolonged courses of antibiotics are associated with adverse drug events, high costs and escalating prevalence of antibiotic-resistant bacteria (10). The optimal duration of therapy for a bloodstream infection would be long enough to effectively eradicate infection and
Bacterial bloodstream infections are common among patients
ing in incidence due to highly invasive care, use of immunosuppressive
medications and interventions, and a tendency to admit patients with
multiple comorbid illnesses ( 3 ). These infections are associated with
up to two- to threefold increase in overall mortality, and up to $35 \%$
attributable mortality $(4,5)$. Prompt initiation of adequate antibiotic
therapy may be one of the most important predictors of patient sur-
vival $(6,7)$, while a delay in adequate therapy (a frequent occurrence

${ }^{1}$ Department of Medicine, University of Toronto; ${ }^{2}$ Department of Critical Care Medicine, Sunnybrook Health Sciences Centre, University of Toronto; ${ }^{3}$ Trauma, Emergency and Critical Care Research Program, Sunnybrook Research Institute; ${ }^{4}$ Department of Pharmacy, Sunnybrook Health Sciences Centre; ${ }^{5}$ Division of Infectious Diseases, Sunnybrook Health Sciences Centre, University of Toronto, Toronto, Ontario Correspondence: Dr Nick Daneman, Division of Infectious Diseases, Sunnybrook Health Sciences Centre, University of Toronto, 2075 Bayview Avenue, Toronto, Ontario M4N 2M5. Telephone 416-480-6100 ext 2791, fax 416-480-5808, e-mail nick.daneman@sunnybrook.ca 
prevent relapse, while short enough to limit adverse effects including Clostridium difficile infection, avoid secondary infection with opportunistic bacterial or fungal pathogens, and minimize selective pressure for antibiotic resistance $(11,12)$. Randomized controlled trials of antibiotic duration for several organ system infections (such as pneumonia, pyelonephritis and intra-abdominal infection) have demonstrated that treatment duration can be shortened to one week or less without compromising patient outcomes (13-16). Limited prospective $(17,18)$ and retrospective (19) evidence suggests that a shorter course of antimicrobial therapy may also be sufficient for the subgroup of patients with bacteremia. However, no randomized controlled trials have directly examined the effectiveness of shorter ( $\leq 7$ to 10 days) versus longer-course antimicrobial therapy for critically ill adults with bacteremia. In the absence of evidence, surveys indicate wide variability in physicians' self-reported prescribing practices, with the most common recommendation being 14 days of treatment, but with at least one-half of clinicians recommending shorter courses ( $\leq 10$ days) $(20,21)$.

The objectives of the present retrospective cohort study were to describe the actual current practice of antibiotic treatment duration for bloodstream infections in critically ill patients, to examine patient, pathogen and infectious syndrome factors associated with selection of shortened treatment duration, and to describe the expected time course of clinical resolution among bacteremic patients.

\section{METHODS}

\section{Study setting}

The present study was conducted at Sunnybrook Health Sciences Centre (SHSC), a large, university-affiliated tertiary care institution located in Toronto, Ontario. SHSC houses six ICUs, which admit a wide spectrum of critically ill patients (total of 82 beds). The three level III ICUs that care for mechanically ventilated patients include a medical-surgical ICU, a cardiovascular ICU and a regional burns centre; the three level II ICUs include a neurosurgical unit, a coronary care unit and a medical-surgical satellite unit. The Sunnybrook Research Ethics Board approved the present study.

\section{General study design and patient selection criteria}

All consecutive patients with a positive blood culture collected in any of the ICUs between March 1, 2010 and March 31, 2011, were considered for inclusion in the cohort. Blood cultures were performed in the SHSC microbiology laboratory using the BACTEC 9240 blood culture system (Becton Dickinson Diagnostic Instrument Systems, USA) (22) in accordance with Clinical Laboratory Standards Institute guidelines (23). True bacteremia was defined as the presence of a pathogenic organism in one or more blood cultures; as per Centers for Disease Control and Prevention (Georgia, USA) definitions, blood cultures for common contaminant species (including coagulase-negative staphylococci, Corynebacterium species, Micrococcus species, Bacillus species and Propionobacterium species) were excluded, unless the organism was isolated from multiple positive blood culture sets in association with clinical signs or symptoms of infection (24). Repeat bacteremia episodes with any pathogen were excluded to ensure that the cohort consisted of unique patients.

\section{Data sources and data collection}

Eligible patients were identified using the Stewardship Program Integrated Resource Information Technology (SPIRIT) database. This relational database, described previously, is automatically populated by health level 7 (HL7) messages from SHSC microbiology, pharmacy and electronic patient care databases, for all admitted patients $(25,26)$. The database is stored on a secure server, and includes comprehensive information on microbiology cultures and pharmacological treatments (including timing and details of anti-infective treatments), as well as limited clinical, administrative and laboratory variables. For each patient determined to meet the definition of true bacteremia, one author $(\mathrm{TH})$ performed an additional review of the entire paper-based and electronic medical record to confirm eligibility. Chart review data were entered into Excel (Microsoft Corporation, USA), merged with SPIRIT data and then transferred to SAS statistical software for analysis (version 9.3, SAS Inc, USA).

The data abstracted for each patient included age, sex, admission category (medical surgical, trauma or burns), dates of hospital admission and discharge, dates of ICU admission and discharge, comorbid medical illnesses (hematological or solid organ malignancies, transplantation, diabetes mellitus, congestive heart failure, obstructive airways disease, chronic renal failure, cirrhosis, HIV, pregnancy, postpartum $<42$ days and immunosuppressive medications), source of index blood cultures (peripheral, arterial or venous catheter, or both), date of blood culture collection, date of blood culture positivity, identity of organism(s), infectious syndrome/source of bacteremia, start date of adequate antimicrobial therapy, stop date of adequate antimicrobial therapy, breakthrough bacteremia after $48 \mathrm{~h}$ of therapy, date of intravascular catheter removal (if applicable), and date and nature of other source control procedure(s) such as percutaneous or surgical drainage of infected fluid.

Outcome data that were extracted included relapse of bacteremia within 30 days of completion of therapy, relapse of infectious syndrome within 30 days, secondary infection with other pathogen(s) or infectious syndrome(s) during therapy, incident infection with $\mathrm{C}$ difficile during hospital stay and in-hospital mortality.

To determine the time course of clinical recovery, a panel of clinical and laboratory parameters were extracted from the date of collection of the positive blood culture (day 0 ) until day 14 , including temperature, heart rate, mean arterial pressure, central venous pressure, vasopressor requirement, fraction of inspired oxygen (\%), arterial partial pressure of oxygen, Glasgow coma score, urine output and need for hemodialysis, white blood cell count, platelet count, bilirubin and creatinine. A subset of these parameters were used to calculate the composite multiple organ dysfunction score (MODS) (27).

\section{Definitions}

Sources of bacteremia were defined as vascular catheter-related, respiratory, abdominal, urinary tract, soft tissue/surgical wound or 'other' when supported by documented clinical, radiographic or additional culture data. A prespecified definition for patients with deep foci of infection with a well-established requirement for prolonged courses of antibiotic therapy included those with infective endocarditis, endovascular infections, prosthetic material/foreign body infections, osteomyelitis, discitis, epidural abscess, septic arthritis and mediastinitis. If the source of infection was neither documented by the treating physicians nor apparent on comprehensive review of records, the source was classified as unknown.

Antimicrobial therapy was deemed to be adequate when at least one antibiotic active in vitro on all organisms identified in the index positive blood culture was administered in the proper dosage. Adequate antimicrobial therapy was considered to be continuous when no interruption in therapy $>24$ h occurred, unless mandated by renal or hepatic dose adjustment. The total duration of antibiotic therapy was considered to be the duration of continuous adequate antimicrobial therapy after the date of index blood culture collection. Duration of antibiotic therapy was dichotomized as short ( $\leq 10$ days) or long ( $>10$ days) for some analyses, based on inspection of the median treatment duration in the cohort.

Antibiotic-free days by 28 days was calculated as the number of days free of antibiotics at 28 days after the antibiotic was started following bacteremia diagnosis. If the patient died within 28 days of antibiotic initiation, the antibiotic-free days were assigned to be 0 . For those who did not receive any antibiotic treatment, the antibiotic-free days were either assigned as 28 if the patient survived to 28 days post bacteremia diagnosis, or were assigned as 0 if they died within 28 days from bacteremia diagnosis. Data from patients who were transferred and receiving antibiotics at the time of transfer were considered to be missing.

\section{Statistical analysis}

The distribution of antibiotic treatment durations (defined above) was plotted, and patients were segregated into five groups according to 
TABLE 1

Underlying infection syndromes and culprit pathogens among the 100 bacteremic episodes

\begin{tabular}{|c|c|c|c|c|c|c|c|c|c|}
\hline Culprit pathogens & Unknown & $\begin{array}{l}\text { Vascular } \\
\text { catheter }\end{array}$ & Respiratory & Abdominal & Urinary tract & $\begin{array}{l}\text { Soft tissuel } \\
\text { wound }\end{array}$ & Other & $\begin{array}{c}\text { Foci requiring } \\
\text { prolonged } \\
\text { treatment }\end{array}$ & All episodes \\
\hline All episodes & 31 & 23 & 17 & 5 & 7 & 3 & 3 & 11 & 100 \\
\hline Gram-positive cocci & 14 & 19 & 6 & 1 & 1 & 3 & 2 & 8 & 54 \\
\hline MSSA & 6 & 1 & 3 & & & 1 & 1 & 3 & 15 \\
\hline MRSA & & & & & & 1 & & 2 & 3 \\
\hline CNST & 3 & 11 & & & & & & & 14 \\
\hline Enterococci & 4 & 7 & 2 & 1 & 1 & & & 1 & 16 \\
\hline Streptococci & 1 & & 1 & & & 1 & 1 & 2 & 6 \\
\hline Gram-negative bacilli & 19 & 4 & 16 & 5 & 6 & 0 & 1 & 3 & 55 \\
\hline Escherichia coli & 4 & & 2 & & 4 & & & & 10 \\
\hline Enterobacter species & 8 & 2 & 1 & & & & & 1 & 12 \\
\hline Pseudomonas species & 3 & & 4 & 1 & 1 & & & 1 & 10 \\
\hline Acinetobacter species & & & 1 & & & & & & 1 \\
\hline Klebsiella species & 4 & & 4 & 4 & 1 & & 1 & & 14 \\
\hline $\begin{array}{l}\text { Stenotrophomonas } \\
\text { species }\end{array}$ & & & 2 & & & & & & 2 \\
\hline Serratia species & & 1 & 1 & & & & & 1 & 3 \\
\hline Proteus species & & 1 & & & 1 & & & & 2 \\
\hline Providencia species & & & 1 & & & & & & 1 \\
\hline Polymicrobial & 2 & & 4 & 1 & 1 & & & & 8 \\
\hline
\end{tabular}

Data presented as n. CNST Coagulase-negative staphylococci; MRSA Methicillin-resistant Staphylococcus aureus; MSSA Methicillin-sensitive S aureus

outcome status in relation to treatment (died before receiving treatment, died during treatment course, discharged/transferred during treatment course, died in hospital after treatment course, or completed treatment course and survived to hospital discharge). A sample size of 85 patients was required to estimate the proportion of patients receiving shorter duration therapy to within $\pm 11 \%$ precision, assuming a true proportion of $50 \%$ (alpha $=0.05)$.

To examine factors predicting the selection of shorter ( $\leq 10$ days) versus longer ( $>10$ days) antibiotic treatment courses, it was necessary to exclude patients who died before receiving therapy, as well as those who died or were discharged/transferred from hospital within 10 days while still receiving antibiotics. In this manner, immortality time bias was accounted for by excluding patients whose duration of therapy was determined by death or discharge rather than antibiotic decisionmaking by the clinical team. Among the remaining patients, univariate statistics were used to test for an association between individual host, pathogen or syndrome characteristics and the likelihood of receiving shorter ( $\leq 10$ days) or longer ( $>10$ days) therapy. The Wilcoxon rank-sum test was applied for continuous predictor variables; Fisher's exact test was applied for tests of proportions. Similar testing was performed in the same subcohort to test the association of treatment duration (now as the predictor variable) with clinical outcomes (relapse of bacteremia, relapse of infectious syndrome, hospital mortality, $\mathrm{C}$ difficile infection and secondary bacterial/fungal bloodstream infection). These analyses were considered to be exploratory given the selection bias inherent in the decision to provide longercourse therapy. In a parallel analysis, all patients (including nonsurvivors) were retained and examined for the association of host, pathogen and syndrome characteristics with the number of antibiotic-free days as calculated above. Wilcoxon rank-sum tests and Kruskal Wallis tests were performed for binary and categorical candidate predictors; Spearman correlation coefficients were calculated for continuous candidate predictors.

For descriptive analyses of the time profile of clinical response, individual components of the MODS were considered to be normal if missing on a given day; if all components were missing, the MODS was considered to be missing (27). If temperature was missing on a single day, it was imputed from the previous day's temperature. Additional statistical modelling was performed for temperature and vasopressor requirements. Temperature was modelled as a continuous variable, using repeated measures analysis with an autoregressive correlation structure to analyze the time effect and the change in slope after seven days. Vasopressor use was modelled as a binary variable, using generalized linear mixed models with an autoregressive correlation structure to analyze the time effect and the change in slope after seven days.

\section{Patient characteristics}

\section{RESULTS}

Exactly 100 unique patients with a first episode of bloodstream infection in critical care were identified during the study period. The majority of these blood cultures were collected more than $48 \mathrm{~h}$ after hospital admission (83 of 100) and more than $48 \mathrm{~h}$ after ICU admission ( 66 of 100$)$. The mean $( \pm$ SD) age of these patients was $66 \pm 17$ years, the majority $(71 \%)$ were male and they encompassed a wide spectrum of admission categories (39\% medical, $40 \%$ surgical, 13\% trauma and $8 \%$ burns). A majority (73\%) of patients had one or more comorbid medical illnesses before admission, the most common being diabetes mellitus $(25 \%)$, solid organ malignancy $(17 \%)$, congestive heart failure $(13 \%)$, chronic obstructive pulmonary disease (12\%), immunosuppressive medications ( $8 \%)$, asthma (6\%) and hematological malignancy $(6 \%)$.

\section{Pathogen characteristics}

A total of 109 infecting organisms were isolated from the index positive blood cultures, with nearly equal numbers of Gram-positive cocci $(n=54)$ and Gram-negative bacilli $(n=55)$. There were 49 patients with monomicrobial Gram-positive bacteremia, 43 patients with monomicrobial Gram-negative bacteremia and eight patients with polymicrobial bacteremia (seven with two organisms and one with three organisms). The most frequently isolated organisms causing bloodstream infection were Staphylococcus aureus (18 patients), Enterococcus species (16 patients), Klebsiella species (14 patients), coagulase-negative staphylococci (14 patients), Enterobacter species (12 patients), Escherichia coli (10 patients) and Pseudomonas species (10 patients) (Table 1). 


\section{TABLE 2}

Patient, pathogen and syndrome characteristics among patients with bloodstream infections for which physicians selected shorter course ( $\leq 10$ days) versus longer course (>10 days) antibiotic therapy*

\begin{tabular}{|c|c|c|c|}
\hline Characteristic & $\leq 10$ days & $>10$ days & $\mathbf{P}^{\dagger}$ \\
\hline Cases, n & $19^{*}$ & 53 & \\
\hline Age, years, mean $\pm \mathrm{SD}$ (range) & $\begin{array}{l}65.6 \pm 19.2 \\
(17.8 \text { to } 85)\end{array}$ & $\begin{array}{c}63.3 \pm 17.1 \\
(26.7 \text { to } 89.2)\end{array}$ & 0.47 \\
\hline Male sex & $16(84)$ & $38(72)$ & 0.36 \\
\hline \multicolumn{4}{|l|}{ Admission category } \\
\hline Medical & $8(42)$ & $18(34)$ & 0.50 \\
\hline Surgical & $6(32)$ & $22(42)$ & \\
\hline Trauma & $4(21)$ & $6(11)$ & \\
\hline Burn & $1(5)$ & $7(13)$ & \\
\hline \multicolumn{4}{|l|}{ Comorbid conditions } \\
\hline Hematological malignancy & $1(5)$ & $2(4)$ & 1.00 \\
\hline Solid organ malignancy & $4(21)$ & $6(11)$ & 0.44 \\
\hline Organ transplant & $0(0)$ & $1(2)$ & 1.00 \\
\hline Diabetes mellitus & $4(21)$ & $13(25)$ & 1.00 \\
\hline Congestive heart failure & $0(0)$ & $8(15)$ & 0.10 \\
\hline COPD & $2(11)$ & $5(9)$ & 1.00 \\
\hline Asthma & $3(16)$ & $2(4)$ & 0.11 \\
\hline $\begin{array}{l}\text { Renal insufficiency on } \\
\text { dialysis }\end{array}$ & $0(0)$ & $5(9)$ & 0.32 \\
\hline Cirrhosis & $0(0)$ & $1(2)$ & 1.00 \\
\hline HIV & $0(0)$ & $0(0)$ & N/A \\
\hline Steroid/immunosuppression & 2 (11) & $4(8)$ & 0.65 \\
\hline Pregnant & $0(0)$ & $0(0)$ & N/A \\
\hline Postpartum (<42 days) & $0(0)$ & $1(2)$ & 1.00 \\
\hline \multicolumn{4}{|l|}{ Source of positive blood culture } \\
\hline Peripheral vein & $6(32)$ & $21(40)$ & 0.70 \\
\hline Central venous or arterial line & $9(47)$ & $17(32)$ & \\
\hline Both & $2(11)$ & $7(13)$ & \\
\hline Unknown & $2(11)$ & $8(15)$ & \\
\hline \multicolumn{4}{|l|}{ Syndrome } \\
\hline Unknown & $4(21)$ & $12(23)$ & 1.00 \\
\hline Vascular catheter & $10(53)$ & $8(15)$ & 0.004 \\
\hline Respiratory & $1(5)$ & $13(25)$ & 0.09 \\
\hline Abdominal & $1(5)$ & $3(6)$ & 1.00 \\
\hline Urinary tract & $3(16)$ & $2(4)$ & 0.11 \\
\hline Soft tissue/wound & $0(0)$ & $3(6)$ & 0.56 \\
\hline Other & $0(0)$ & $1(2)$ & 1.00 \\
\hline $\begin{array}{l}\text { Foci requiring prolonged } \\
\text { therapy }\end{array}$ & $0(0)$ & $11(21)$ & 0.03 \\
\hline ICU-acquired bacteremia & $13(68)$ & $33(63)$ & 0.63 \\
\hline $\begin{array}{l}\text { Receiving antibiotics at time of } \\
\text { bacteremia detection }\end{array}$ & $7(36.8)$ & $19(35.8)$ & 1.00 \\
\hline MODS at bacteremia detection & 6.6 & 7.6 & 0.23 \\
\hline $\begin{array}{l}\text { Breakthrough bacteremia after } \\
\geq 48 \mathrm{~h}\end{array}$ & $0(0)$ & $11(23)$ & 0.03 \\
\hline Gram-positive cocci & $12(63)$ & $28(53)$ & \\
\hline Staphylococcus aureus & $0(0)$ & $13(25)$ & 0.02 \\
\hline $\begin{array}{l}\text { Coagulase-negative } \\
\text { staphylococci }\end{array}$ & $8(42)$ & $2(4)$ & 0.0002 \\
\hline Enterococcus species & $3(16)$ & $9(17)$ & 1.00 \\
\hline Streptococcus species & $1(5)$ & $4(8)$ & 1.00 \\
\hline Gram-negative bacilli & $8(42)$ & $30(57)$ & \\
\hline Escherichia coli & $3(16)$ & $4(8)$ & 0.37 \\
\hline Enterobacter species & $1(5)$ & $7(13)$ & 0.67 \\
\hline Pseudomonas species & $0(0)$ & $8(15)$ & 0.10 \\
\hline
\end{tabular}

Continued in next column
TABLE 2 - CONTINUED

Patient, pathogen, and syndrome characteristics among patients with bloodstream infections for which physicians selected shorter course ( $\leq 10$ days) versus longer course ( $>10$ days) antibiotic therapy*

\begin{tabular}{lccc}
\hline Characteristic & $\mathbf{\leq 1 0}$ days & $>$ 10 days & $\mathbf{P}^{\dagger}$ \\
\hline Acinetobacter species & $0(0)$ & $1(2)$ & 1.00 \\
Klebsiella species & $2(11)$ & $5(9)$ & 1.00 \\
Stenotrophomonas species & $0(0)$ & $1(2)$ & 1.00 \\
Serratia species & $0(0)$ & $3(6)$ & 0.56 \\
Proteus species & $1(5)$ & $1(2)$ & 0.46 \\
Providencia species & $1(5)$ & $0(0)$ & 0.26 \\
\hline
\end{tabular}

Data presented as $n$ (\%) unless otherwise specified. *Patients dying on therapy $\leq 10$ days were excluded from the present analysis; ${ }^{\dagger}$ Fischer's exact tests for proportions, Wilcoxon rank-sum tests for continuous variables. COPD Chronic obstructive pulmonary disease; ICU Intensive care unit; MODS Multiple organ dysfunction score; N/A Not applicable

Infection syndrome characteristics

The most common perceived sources of bacteremia were vascular catheter infection $(n=23)$, lower respiratory infection $(n=17)$, urinary tract infection $(n=7)$, abdominal infection $(n=5)$ and soft tissue/ wound infection $(n=3)$. Collectively, there were 11 cases in which patients had deep foci of infection with established requirement for prolonged courses of antibiotic therapy (two with epidural abscesses, two with septic arthritis, two with infective endocarditis, and one each with an infected vascular graft, sternal osteomyelitis, undrainable intra-abdominal abscess and mediastinitis). The source of infection remained unknown to both the treating team and chart reviewer in nearly one-third of cases (31\%) (Table 1 ).

Duration of antibiotic therapy

The median duration of microbiologically adequate antibiotic therapy use to treat these bloodstream infections was 11 days (interquartile range [IQR] 4.5 to 17 days), but was highly variable (range zero to 60 days). After excluding patients who died or were discharged within 10 days and before completing therapy or those with deep foci for which prolonged therapy is well established, the median treatment duration was 13 days (IQR 10 to 17 days; range zero to 34 days). Six patients died before receiving treatment for their bacteremia, 22 died during their course for bacteremia, and 12 were discharged from hospital or transferred while still receiving treatment. Of the 60 patients who completed their treatment course in hospital, 11 died subsequently to completion of treatment and 49 survived to discharge. The full distribution of treatment durations (segregated according to outcome status) is illustrated in Figure 1.

Association of antibiotic treatment duration (and antibiotic-free days) with patient, pathogen and syndrome characteristics

The impact of patient, pathogen and syndrome characteristics on selection of shorter ( $\leq 10$ days) and longer ( $>10$ days) antibiotic treatment courses is summarized in Table 2; the impact of these characteristics on the number of antibiotic-free days is summarized in Table 3. Longer durations of antibiotic treatment (and fewer antibiotic-free days) were associated with foci having established requirements for prolonged treatment, and $S$ aureus bacteremia. There were also trends toward longer duration treatment for underlying respiratory tract foci and Pseudomonas bacteremias. Conversely, shorter durations of antibiotic treatment (and more antibiotic-free days) were associated with vascular catheter-related bloodstream infections and coagulase-negative staphylococcal bacteremia. Breakthrough bacteremia despite $48 \mathrm{~h}$ of effective treatment was associated with longer treatment duration, but not with fewer antibiotic-free days (likely due to survivor bias).

The median durations of antibiotic treatment provided for the most common causes of bacteremia included seven days (IQR two to 


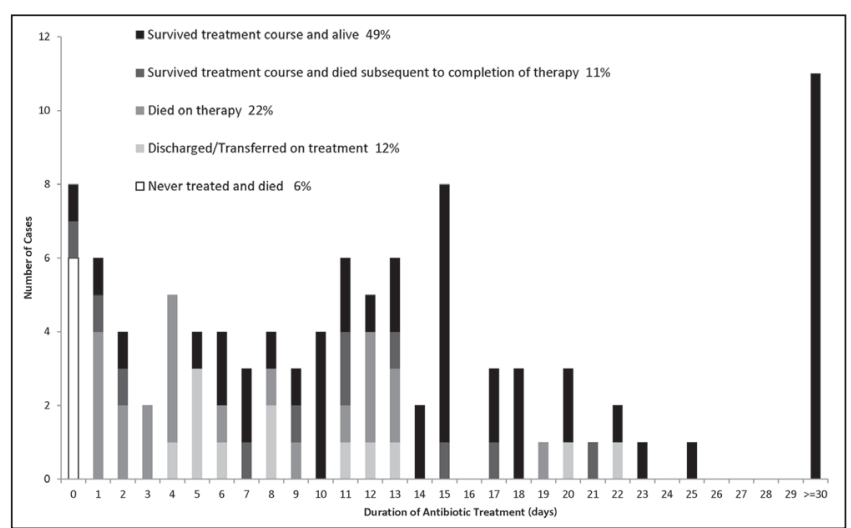

Figure 1) Duration of antibiotic therapy. The duration of antibiotic therapy is displayed for each of the 100 patients with bacteremia. The total duration of antibiotic therapy was defined by the number of consecutive days of antibiotics active against the culprit pathogen, after the index date of blood culture collection. The bars are segmented according to the patient's survival status, and timing of death in relation to initiation and completion of antibiotic therapy. This figure illustrates the potential for immortal time bias among patients who have the opportunity for longer treatments

13 days) for vascular catheter-related bacteremia, 15 days (IQR 11 to 17 days) for respiratory sources, 15 days (IQR 10 to 15 days) for abdominal sources, eight days (IQR four to 13 days) for urinary tract sources, 20 days (IQR 13 to 22 days) for soft tissue/wound sources and eight days (IQR three to 13 days) for bacteremia of unknown source.

Patient outcomes in relation to duration of therapy

The overall hospital mortality rate for the cohort of 100 critically ill bacteremic patients was 39\%. Six deaths occurred before receiving treatment, all with fulminant sepsis on the day their positive blood culture was collected and before the blood culture positivity was detected in the microbiology laboratory. An additional 22 patients died while still receiving treatment, including 15 before day 10 , six between days 11 to 14 and one after day 14 . Only a minority of deaths (11 of 39 [28\%]) occurred after completion of antibiotic therapy. For the cohort of 72 patients who survived to the potential decision point of shorter versus longer treatment, downstream outcomes were similar between the two groups (Table 4).

Time profile of clinical response

There was a significant decrease in temperatures in the first seven days (reduction of $0.16^{\circ} \mathrm{C}$ per day, $95 \%$ CI 0.11 to $0.20 ; \mathrm{P}<00001$ ), but no significant change in temperatures after seven days (mean decrease of $0.02^{\circ} \mathrm{C}$ per day, $95 \% \mathrm{CI}-0.02$ to $0.06 ; \mathrm{P}=0.31$ ) (Figure 2). Trends in white blood cell counts and MODS are displayed in Figures 3 and 4. Vasopressor use declined over the 14-day period (OR 0.9 per day, $95 \%$ CI 0.93 to $0.98 ; \mathrm{P}=0.018$ ), with no significant plateau after seven days (Figure 5).

\section{DISCUSSION}

In the absence of previous randomized controlled trials to inform clinical practice, critically ill patients with bloodstream infections are being treated with highly variable and, in some cases, prolonged durations of antibiotic therapy (21). Selection of treatment duration does not appear to be driven by host demographics and comorbidities, but does appear to be influenced by the culprit pathogen and infectious syndrome. Longer antibiotic treatment durations are provided for respiratory tract infections, and for etiologies including $S$ aureus and Pseudomonas species. Shorter-duration therapy is more commonly selected for vascular catheter-associated bacteremias, especially those due to coagulase-negative staphylococci.

The present retrospective cohort study of actual clinical practice corroborates a national survey of Canadian infectious diseases and

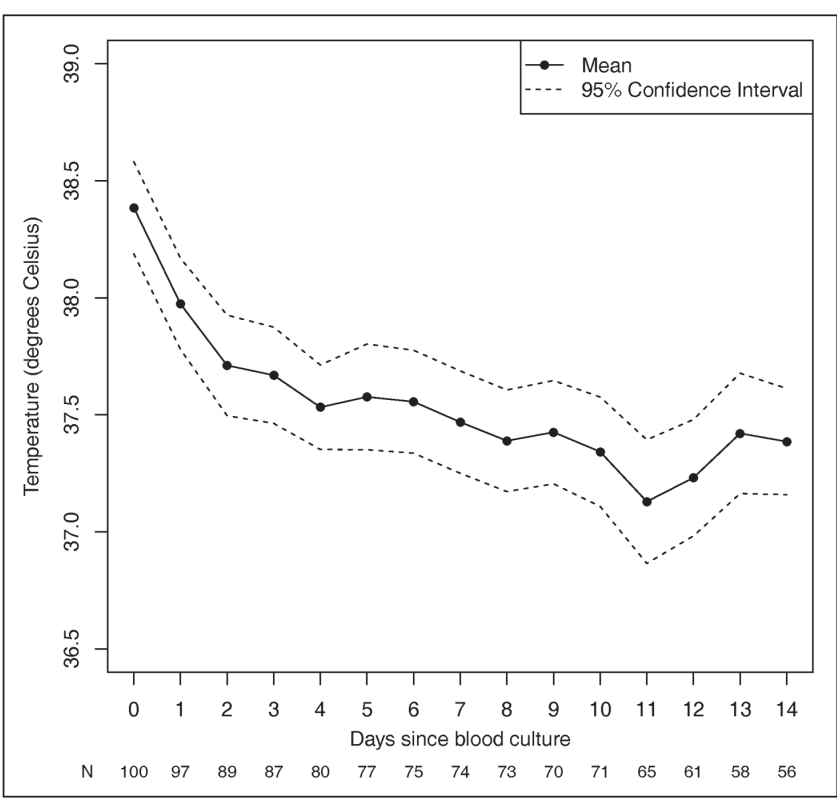

Figure 2) Time profile of temperatures among critically ill patients with bloodstream infection. There was a significant decrease in temperatures in the first seven days (reduction of $0.16^{\circ} \mathrm{C}$ per day, $95 \%$ CI 0.11 to 0.20 ; $P<00001)$, but no significant change in temperatures after seven days (mean decrease of $0.02^{\circ} \mathrm{C}$ per day, $95 \% \mathrm{CI}-0.02$ to $0.06 ; \mathrm{P}=0.31$ )

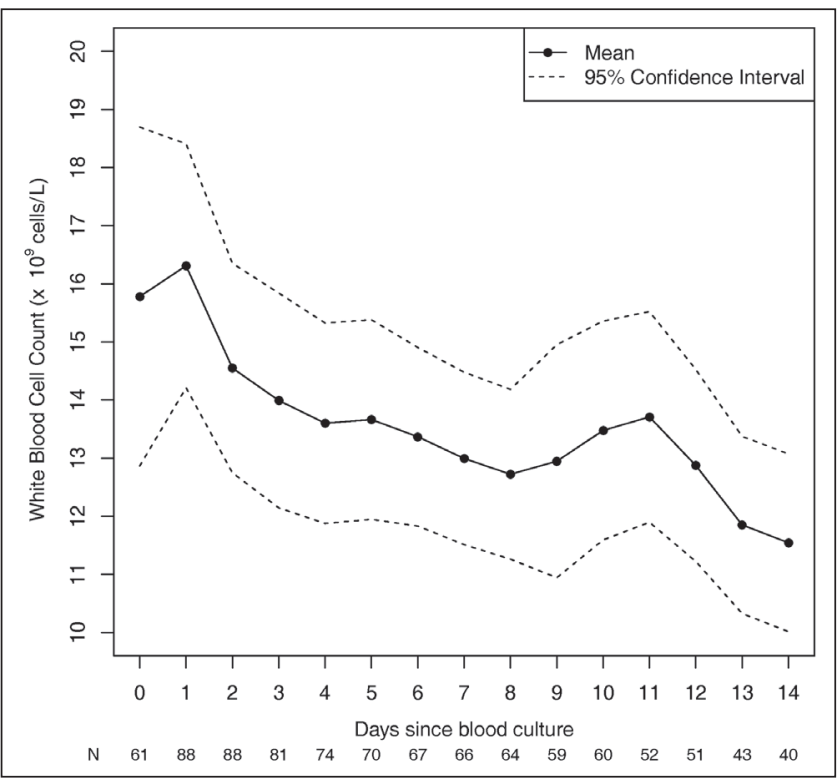

Figure 3) Time profile of white blood cell counts among critically ill patients with bloodstream infection. White blood cell count declined over the 14-day interval, although there was substantial variation in time profiles among individuals

critical care physicians that identified wide practice heterogeneity in antibiotic treatment duration recommendations for bacteremia in critically ill patients (21). Such practice variability is not surprising given the absence of previous research on this topic, and the lack of guidance in pneumonia, urinary tract, soft tissue and intra-abdominal infection guidelines about optimal duration of therapy for the subset of patients with bacteremia (28-32). Similarly, the present study confirms previous survey findings that clinicians most commonly favour prolonged treatment durations (21). Therefore, if future research establishes that shorter-duration therapy is as effective as longer-duration treatment for bacteremia (as has now been documented for multiple infectious 
TABLE 3

Association of patient, pathogen and syndrome characteristics with number of antibiotic-free days in the 28 days following bacteremia diagnosis*

\begin{tabular}{|c|c|c|c|}
\hline Characteristic & $\begin{array}{l}\text { Antibiotic-free days* with versus } \\
\text { without characteristic, median (IQR) }\end{array}$ & $\begin{array}{l}\text { Patients with/ } \\
\text { without characteristic, } \mathrm{n}\end{array}$ & $\mathbf{P}^{\dagger}$ \\
\hline Age & $-0.249^{\ddagger}$ & 88 & 0.02 \\
\hline \multicolumn{4}{|l|}{ Sex } \\
\hline Female & $0(0-13)$ & 26 & 0.30 \\
\hline Male & $5.5(0-17)$ & 62 & \\
\hline \multicolumn{4}{|l|}{ Admission category } \\
\hline Medical & $0(0-13)$ & 34 & 0.17 \\
\hline Surgical & $0(0-15)$ & 34 & \\
\hline Trauma & $13(5-18.5)$ & 12 & \\
\hline Burns & $9.5(5.5-13)$ & 8 & \\
\hline \multicolumn{4}{|l|}{ Comorbid conditions (no versus yes) } \\
\hline Hematological malignancy & $5.5(0-15)$ versus $0(0-13)$ & $82 / 6$ & 0.53 \\
\hline Solid malignancy & $6(0-15)$ versus $0(0-18)$ & $75 / 13$ & 0.81 \\
\hline Organ transplantation & $3(0-15)$ versus $13(13-13)$ & $87 / 1$ & 0.54 \\
\hline Diabetes mellitus & $6(0-15)$ versus $0(0-15)$ & $67 / 21$ & 0.60 \\
\hline Congestive heart failure & $8(0-17)$ versus $0(0-0)$ & $75 / 13$ & 0.03 \\
\hline Chronic obstructive pulmonary disease & $5.5(0-15)$ versus $0(0-11)$ & $78 / 10$ & 0.42 \\
\hline Asthma & $3(0-14)$ versus $18(0-26)$ & $83 / 5$ & 0.21 \\
\hline Chronic renal failure & $5.5(0-15)$ versus $1.5(0-5)$ & $84 / 4$ & 0.22 \\
\hline Cirrhosis & $5.5(0-15)$ versus $0(0-0)$ & $86 / 2$ & 0.18 \\
\hline Steroids/immunosuppression & $1.5(0-15)$ versus13 (0-17.5) & $80 / 8$ & 0.43 \\
\hline Postpartum $<42$ days & $3(0-15)$ versus $14(14-14)$ & $87 / 1$ & 0.50 \\
\hline \multicolumn{4}{|l|}{ Source of positive blood culture } \\
\hline Peripheral vein & $0(0-13)$ & 34 & 0.19 \\
\hline Central venous or arterial line & $11.5(0-18)$ & 30 & \\
\hline Both & $0(0-13)$ & 12 & \\
\hline Unknown & $1.5(0-12)$ & 12 & \\
\hline \multicolumn{4}{|l|}{ Syndrome/source of bacteremia } \\
\hline Unknown & $0(0-14)$ versus $6(0-15)$ & 27 & 0.48 \\
\hline Vascular catheter-related & $14.5(0-22)$ versus $0(0-13)$ & 22 & 0.01 \\
\hline Respiratory & $10(0-13)$ versus $0(0-15.5)$ & 16 & 0.76 \\
\hline Abdominal & $13(0-13)$ versus $3(0-15)$ & 5 & 0.80 \\
\hline Urinary tract & $10(0-19)$ versus $3(0-15)$ & 5 & 0.56 \\
\hline Soft tissue/wound & 15 (15-15) versus $3(0-15)$ & 1 & 0.44 \\
\hline Other & $0(0-0)$ versus $5.5(0-15)$ & 2 & 0.21 \\
\hline Focus requiring prolonged treatment & $9(0-16)$ versus $0(0-0)$ & $10 / 78$ & 0.002 \\
\hline Receiving antibiotics at time of bacteremia detection & $5(0-15)$ versus $0(0-13)$ & $55 / 33$ & 0.88 \\
\hline MODS at bacteremia detection & $-0.41^{\ddagger}$ & 88 & $<0.001$ \\
\hline Breakthrough bacteremia $\geq 48 \mathrm{~h}$ & $0(0-15)$ versus $10(0-11)$ & $77 / 11$ & 0.73 \\
\hline \multicolumn{4}{|l|}{ Gram-positive cocci } \\
\hline Staphylococcus aureus & $0(0-9)$ versus $6.5(0-16.5)$ & 12 & 0.07 \\
\hline Coagulase-negative staphylococci & $18(0-26)$ versus $0(0-13)$ & 13 & 0.02 \\
\hline Enterococcus species & $13(0-15.5)$ versus $0(0-14)$ & 16 & 0.37 \\
\hline Streptococcus species & $13(0-15)$ versus $3(0-15)$ & 5 & 0.62 \\
\hline \multicolumn{4}{|l|}{ Gram-negative bacilli } \\
\hline Escherichia coli & $0(0-13)$ versus $5(0-15)$ & 9 & 0.76 \\
\hline Enterobacter species & $5(0-13)$ versus $3(0-15)$ & 11 & 0.85 \\
\hline Pseudomonas species & 0 (0-10) versus $6(0-15)$ & 10 & 0.20 \\
\hline Acinetobacter species & $13(13-13)$ versus $3(0-15)$ & 1 & 0.54 \\
\hline Klebsiella species & $10.5(0-14)$ versus $1.5(0-15)$ & 10 & 0.80 \\
\hline Stenotrophomonas species & $0(0-0)$ versus $5.5(0-15)$ & 2 & 0.18 \\
\hline Serratia species & $3(0-5)$ versus $6(0-15)$ & 3 & 0.59 \\
\hline Proteus species & $14.5(7-22)$ versus $1.5(0-15)$ & 2 & 0.25 \\
\hline Providencia species & 18 (18-18) versus $3(0-15)$ & 1 & 0.22 \\
\hline
\end{tabular}

*Antibiotic-free days by 28 days was calculated as the number of days free of antibiotics at 28 days after the antibiotic was started following bacteremia diagnosis. If the patient died within 28 days of antibiotic initiation the antibiotic-free days were assigned to be 0 ; ${ }^{\dagger} P$ values represent Wilcoxon rank sum tests for binary variables, and Kruskal-Wallis tests for nonbinary categorical variables; †Spearman correlation coefficients were calculated for continuous predictor variables. IQR Interquartile range; MODS Multiple organ dysfunction score 
TABLE 4

Outcomes among patients with bloodstream infections for which physicians selected shorter course ( $\leq 10$ days) versus longer course ( $>10$ days) antibiotic therapy*

\begin{tabular}{llcc}
\hline Outcome & \multicolumn{1}{c}{$\mathbf{S 1 0}$ days } & $>10$ days & $\mathbf{P}^{\dagger}$ \\
\hline Mortality & $5 / 19(26)$ & $13 / 53(25)$ & 1.00 \\
Relapse of bacteremia & $1 / 19(5)$ & $4 / 53(8)$ & 1.00 \\
Relapse of underlying infectious & $1 / 19(5)$ & $3 / 53(6)$ & 1.00 \\
$\quad$ syndrome & & & \\
Clostridium difficile infection & $4 / 19(21)$ & $5 / 53(9)$ & 0.23 \\
Secondary bloodstream infection & $5 / 19(26)$ & $9 / 53(17)$ & 0.50 \\
\hline
\end{tabular}

Data presented as $n / n$ (\%). *Patients dying during therapy $\leq 10$ days were excluded from the analysis; ${ }^{\dagger} P$ values represent Fischer's exact test for proportions, Wilcoxon rank-sum tests for continuous variables

syndromes) (33), there is great potential to both standardize clinical practice and reduce the overall burden of antibiotic use in critical care.

Whereas a previous clinical practice survey (21) revealed very discrete treatment duration recommendations (seven, 10 or 14 days of antibiotics according to the vast majority of clinicians), actual clinical practice is much less discrete and much more complicated. In actual practice, clinicians may be less prone to rely on multiples of five and seven days, and more likely to adapt treatment based on perceived clinical response. In many cases, treatment duration is not decided by the clinician, but by timing of patient outcomes (eg, death, most often occurring while patients were still receiving treatment). Any future randomized controlled trials of duration of therapy will need to consider the large fractions of patients who will not contribute to effective sample size because death precedes antibiotic discontinuation decision making.

The prolonged durations of therapy provided to patients with bacteremic respiratory tract infection may suggest that, although randomized controlled trials have documented that shorter course therapy is sufficient for both community-acquired and ventilator-associated pneumonia, perhaps clinicians are reluctant to generalize these findings to the subgroup of patients with bacteremia $(15,33)$. Conversely, the shorter durations of treatments provided to patients with vascular catheter-associated bacteremia may indicate a willingness to proceed with shorter-course regimens even in the absence of evidence for this focus. Our finding that one-third of bacteremias have unknown sources favours further investigation focusing on bacteremia (rather than individual underlying syndromes) so that this large subset will not be neglected. This approach may be reasonable given that the Canadian survey data suggested that the presence of bacteremia is an important driver of antibiotic treatment duration, and that typically recommended treatment durations are similar for each of the common bacteremic syndromes (21). Other advantages of studying bacteremia are that, by definition, a culprit pathogen(s) is/are identified for all cases; adequacy of antibiotic selection can then be determined for all cases, and if shorter-duration treatment is equivalent to longerduration treatment for bacteremia, results will be more easily generalizable to nonbacteremic infections than vice versa.

The preference for prolonged durations of antibiotics for $S$ aureus likely relates to the capacity of this organism to adhere to a range of host tissues (34), along with the findings of previous observational studies documenting higher rates of metastatic infection with therapy of less than two weeks' duration $(35,36)$. The preference for a shorter duration of therapy for coagulase-negative staphylococcal bacteremias may relate to the limited virulence of these skin colonizers, and their requirement of prosthetic material (such as an in situ vascular catheter) to cause persistent infection (37). It is possible that clinicians may be reluctant to randomly assign patients with $S$ aureus bacteremia to the possibility of short-course therapy, or coagulase-negative staphylococci to the possibility of prolonged treatment. Breakthrough bacteremia beyond $48 \mathrm{~h}$ of effective treatment was also associated with selection of prolonged treatment courses, which is likely appropriate

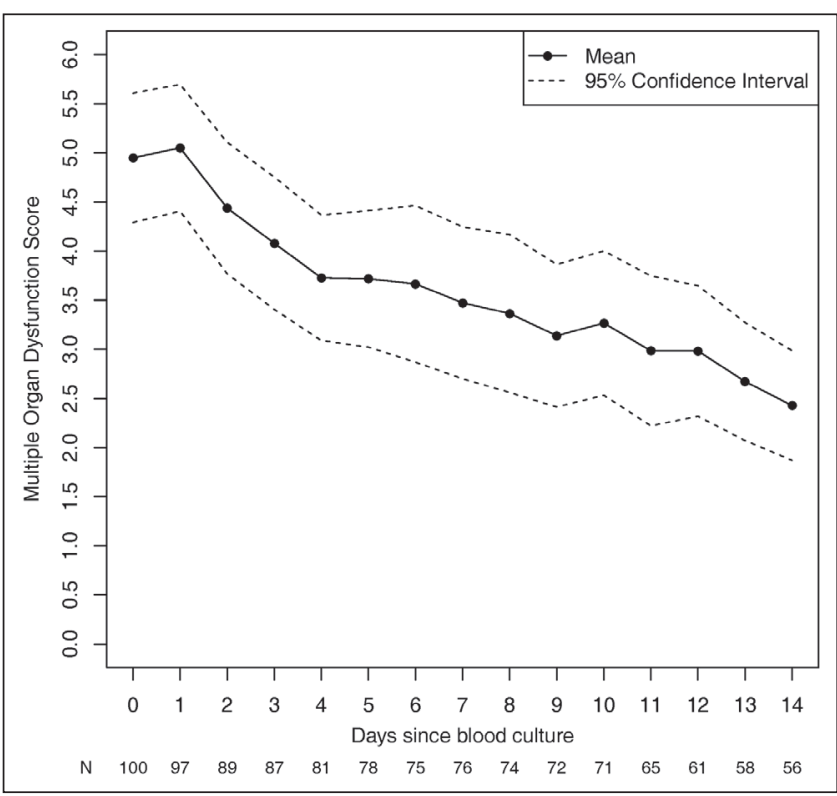

Figure 4) Time profile of multiple organ dysfunction scores among critically ill patients with bloodstream infection. Multiple organ dysfunction scores declined over the 14-day interval, although there was substantial variation in time profiles across individuals

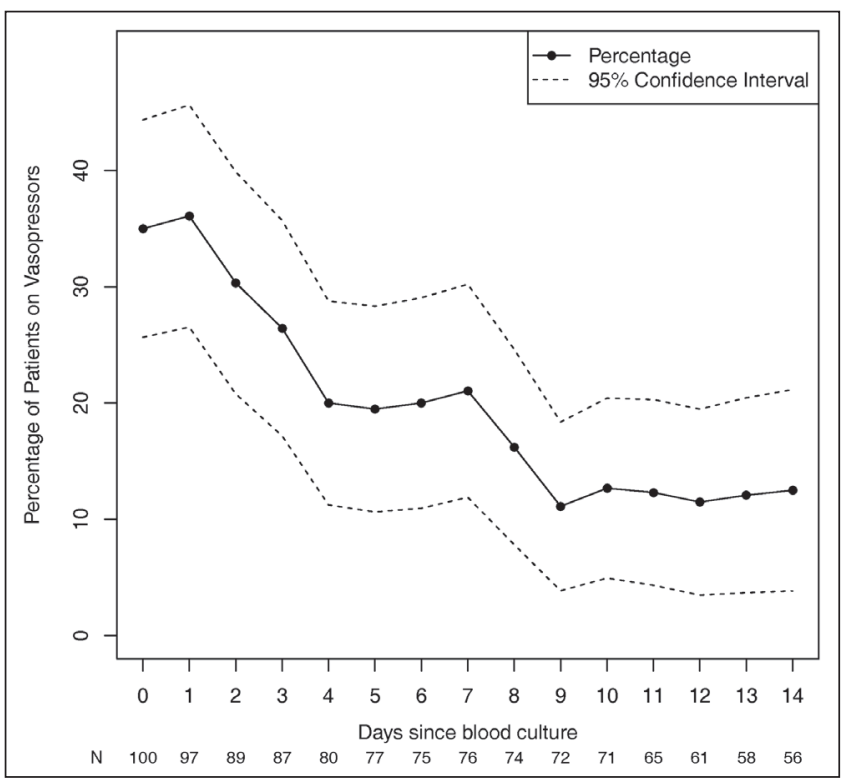

Figure 5) Time profile of vasopressor requirements among critically ill patients with bloodstream infection. Vasopressor use declined over the 14-day period (OR 0.9/day, 95\% CI 0.93 to 0.98; $\mathrm{P}=0.018$ ), with no significant plateau after seven days

given that this phenomenon has been strongly associated with the presence of an undetected intravascular focus or undrained abscess $(38,39)$.

The time profile of clinical response among survivors illustrates the difficulty in determining an individualized antibiotic stop date for critically ill patients with bacteremia. Most parameters exhibited large interindividual variation. The reduction of temperature within the first (but not second week) provides some support for the notion that short-course therapy may be sufficient, but white blood cell counts, MODS scores and vasopressor dependence did not clearly plateau over this time interval. However, there is an expected delay in resolution of 
markers of inflammation even after the inciting infection as been eradicated and, as such, inflammatory biomarkers may often be inappropriate tools to guide duration of antibiotic therapy.

An observational study assessing the impact of treatment duration on patient outcomes is limited by survivor bias (patients must survive long enough to be classified as receiving longer-duration treatment) and bias-by-indication (clinicians systematically select sicker patients to receive longer-duration treatment). However, our primary objectives were to describe antibiotic treatment durations, and the predictors of these treatment durations, rather than the downstream impact on patient outcomes. The heterogeneity of infectious foci and pathogens translated to few patients with any individual type of infection; therefore, our findings should be corroborated by future multicentre research powered to precisely estimate treatment durations within these subgroups.

We found that antibiotic treatment durations exhibit extensive variability, are often prolonged, and appear to be influenced by the underlying pathogen and syndrome. Ultimately, a randomized controlled trial will be essential to provide an evidence basis for this important therapeutic decision. Patients can be randomly assigned at the time of blood culture positivity to fixed short course (eg, seven days) versus fixed longer-course (eg, 14 days) antibiotic therapy. The trial would need to account for the large proportions of early deaths that would not contribute meaningful data to this research question, and would also need to be powered to assess outcomes in individual pathogen, syndrome and patient subgroups. It may be necessary to exclude $S$ aureus bacteremias (due to lack of equipoise for shorterduration treatment) and coagulase-negative staphylococcal vascular catheter-related bacteremias (due to lack of equipoise to support longer duration treatment). Such a trial will be challenging, but will be crucial to define optimal treatment durations that maximize clinical cure of bloodstream infections while minimizing the adverse consequences associated with unnecessary antibiotic use.

ACKNOWLEDGEMENTS: Nick Daneman is supported by a Clinician Scientist Award from the Canadian Institutes of Health Research. Rob Fowler is a Clinician Scientist of the Heart and Stroke Foundation. Additional funding for this study was provided by an Ontario Ministry of Health and Long-term Care Academic Health Sciences Centre Innovation Fund Award. The funding bodies had no role in study design, data collection, data interpretation, manuscript writing or decision to submit for publication.

\section{REFERENCES}

1. Vincent JL, Rello J, Marshall J, et al. International study of the prevalence and outcomes of infection in intensive care units. JAMA 2009;302:2323-9.

2. Valles J, Leon C, varez-Lerma F. Nosocomial bacteremia in critically ill patients: A multicenter study evaluating epidemiology and prognosis. Spanish Collaborative Group for Infections in Intensive Care Units of Sociedad Espanola de Medicina Intensiva y Unidades Coronarias (SEMIUC). Clin Infect Dis 1997;24:387-95.

3. Kumar G, Kumar N, Taneja A, et al. Nationwide trends of severe sepsis in the 21st century (2000-2007). Chest 2011;140:1223-31.

4. Pittet D, Tarara D, Wenzel RP. Nosocomial bloodstream infection in critically ill patients. Excess length of stay, extra costs, and attributable mortality. JAMA 1994;271:1598-601.

5. Renaud B, Brun-Buisson C. Outcomes of primary and catheterrelated bacteremia. A cohort and case-control study in critically ill patients. Am J Respir Crit Care Med 2001;163:1584-90.

6. Garrouste-Org, Timsit JF, Tafflet M, et al. Excess risk of death from intensive care unit-acquired nosocomial bloodstream infections: A reappraisal. Clin Infect Dis 2006;42:1118-26.

7. Kumar A, Roberts D, Wood KE, et al. Duration of hypotension before initiation of effective antimicrobial therapy is the critical determinant of survival in human septic shock. Crit Care Med 2006;34:1589-96.

8. Harbarth S, Garbino J, Pugin J, Romand JA, Lew D, Pittet D. Inappropriate initial antimicrobial therapy and its effect on survival in a clinical trial of immunomodulating therapy for severe sepsis. Am J Med 2003;115:529-35.

9. Ibrahim EH, Sherman G, Ward S, Fraser VJ, Kollef MH. The influence of inadequate antimicrobial treatment of bloodstream infections on patient outcomes in the ICU setting. Chest 2000;118:146-55.

10. Rubinstein E. Short antibiotic treatment courses or how short is short? Int J Antimicrob Agents 2007;30(Suppl 1):S76-S79.

11. Rice LB. The Maxwell Finland Lecture: For the duration-rational antibiotic administration in an era of antimicrobial resistance and Clostridium difficile. Clin Infect Dis 2008;46:491-6.

12. Aarts MA, Brun-Buisson C, Cook DJ, et al. Antibiotic management of suspected nosocomial ICU-acquired infection: Does prolonged empiric therapy improve outcome? Intensive Care Med 2007;33:1369-78

13. Chastre J, Wolff M, Fagon JY, et al. Comparison of 8 vs 15 days of antibiotic therapy for ventilator-associated pneumonia in adults: A randomized trial. JAMA 2003;290:2588-98.

14. Basoli A, Chirletti P, Cirino E, et al. A prospective, double-blind, multicenter, randomized trial comparing ertapenem 3 vs $>$ or $=5$ days

in community-acquired intraabdominal infection. J Gastrointest Surg 2008;12:592-600.

15. Sandberg T, Skoog G, Bornefalk Hermansson A, et al. Ciprofloxacin for 7 versus 14 days in women with acute pyelonephritis: A randomised, open-label and double-blind, placebo-controlled non-inferiority trial. Lancet 2012;380:484-90.

16. Dimopoulos G, Matthaiou DK, Karageorgopoulos DE, et al. Short-versus long-course antibacterial therapy for communityacquired pneumonia: A meta-analysis. Drugs 2008;68:1841-54.

17. Corona A, Bertolini G, Lipman J, Wilson AP, Singer M. Antibiotic use and impact on outcome from bacteraemic critical illness: The BActeraemia Study in Intensive Care (BASIC). J Antimicrob Chemother 2010;65:1276-85.

18. Chowdhary G, Dutta S, Narang A. Randomized controlled trial of 7-day vs. 14-day antibiotics for neonatal sepsis. J Trop Pediatr 2006;52:427-32.

19. Havey TC, Fowler RA, Daneman N. Duration of antibiotic therapy for bacteremia: A systematic review and meta-analysis. Crit Care 2011;15:R267.

20. Corona A, Bertolini G, Ricotta AM, Wilson AP, Singer M. Variability of treatment duration for bacteraemia in the critically ill: A multinational survey. J Antimicrob Chemother 2003;52:849-52.

21. Daneman N, Shore K, Pinto R, Fowler R. Antibiotic treatment duration for bloodstream infections in critically ill patients: A national survey of Canadian infectious diseases and critical care specialists. Int J Antimicrob Agents 2011;38:480-5.

22. Nolte FS, Williams JM, Jerris RC, et al. Multicenter clinical evaluation of a continuous monitoring blood culture system using fluorescent-sensor technology (BACTEC 9240). J Clin Microbiol 1993;31:552-7.

23. Clinical and Laboratory Standards Institute. Principles and Procedures for Blood Cutlures; Approved Guideline M47-A, 2011. Report No.: 27.

24. Horan TC, Andrus M, Dudeck MA. CDC/NHSN surveillance definition of health care-associated infection and criteria for specific types of infections in the acute care setting. Am J Infect Control 2008;36:309-32.

25. Elligsen M, Walker S, Simor A, Daneman N. Prospective audit and feedback of antimicrobial stewardship in critical care: Program implementation, experience, and challenges. Can J Hosp Pharm 2012;65:31-6.

26. Elligsen M, Walker SA, Pinto R, et al. Audit and feedback to reduce broad-spectrum antibiotic use among intensive care unit patients: A controlled interrupted time series analysis. Infect Control Hosp Epidemiol 2012;33:354-61.

27. Marshall JC, Cook DJ, Christou NV, Bernard GR, Sprung CL, Sibbald WJ. Multiple organ dysfunction score: A reliable descriptor of a complex clinical outcome. Crit Care Med 1995;23:1638-52. 
28. Mandell LA, Wunderink RG, Anzueto A, et al. Infectious Diseases Society of America/American Thoracic Society consensus guidelines on the management of community-acquired pneumonia in adults. Clin Infect Dis 2007;44(Suppl 2):S27-S72.

29. American Thoracic Society; Infectious Diseases Society of America. Guidelines for the management of adults with hospital-acquired, ventilator-associated, and healthcare-associated pneumonia. Am J Respir Crit Care Med 2005;171:388-416.

30. Solomkin JS, Mazuski JE, Bradley JS, et al. Diagnosis and management of complicated intra-abdominal infection in adults and children: Guidelines by the Surgical Infection Society and the Infectious Diseases Society of America. Clin Infect Dis 2010;50:133-64.

31. Gupta K, Hooton TM, Naber KG, et al. International clinical practice guidelines for the treatment of acute uncomplicated cystitis and pyelonephritis in women: A 2010 update by the Infectious Diseases Society of America and the European Society for Microbiology and Infectious Diseases. Clin Infect Dis 2011;52:e103-e120.

32. Stevens DL, Bisno AL, Chambers HF, et al. Practice guidelines for the diagnosis and management of skin and soft-tissue infections. Clin Infect Dis 2005;41:1373-406.
33. Havey T, Fowler RA, Daneman N. Duration of antibiotic therapy for bacteremia: A systematic review and meta-analysis. Crit Care 2011;15:R267.

34. Lowy FD. Staphylococcus aureus infections. N Engl J Med 1998;339:520-32.

35. Fowler VG Jr, Sanders LL, Sexton DJ, et al. Outcome of Staphylococcus aureus bacteremia according to compliance with recommendations of infectious diseases specialists: Experience with 244 patients. Clin Infect Dis 1998;27:478-86.

36. Jernigan JA, Farr BM. Short-course therapy of catheter-related Staphylococcus aureus bacteremia: A meta-analysis. Ann Intern Med 1993;119:304-11.

37. Mermel LA, Allon M, Bouza E, et al. Clinical practice guidelines for the diagnosis and management of intravascular catheter-related infection: 2009 update by the Infectious Diseases Society of America. Clin Infect Dis 2009;49:1-45.

38. Hawkins C, Huang J, Jin N, Noskin GA, Zembower TR, Bolon M. Persistent Staphylococcus aureus bacteremia: An analysis of risk factors and outcomes. Arch Intern Med 2007;167:1861-7.

39. Weinstein MP, Reller LB. Clinical importance of "breakthrough" bacteremia. Am J Med 1984;76:175-80. 


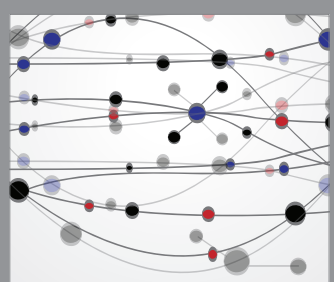

The Scientific World Journal
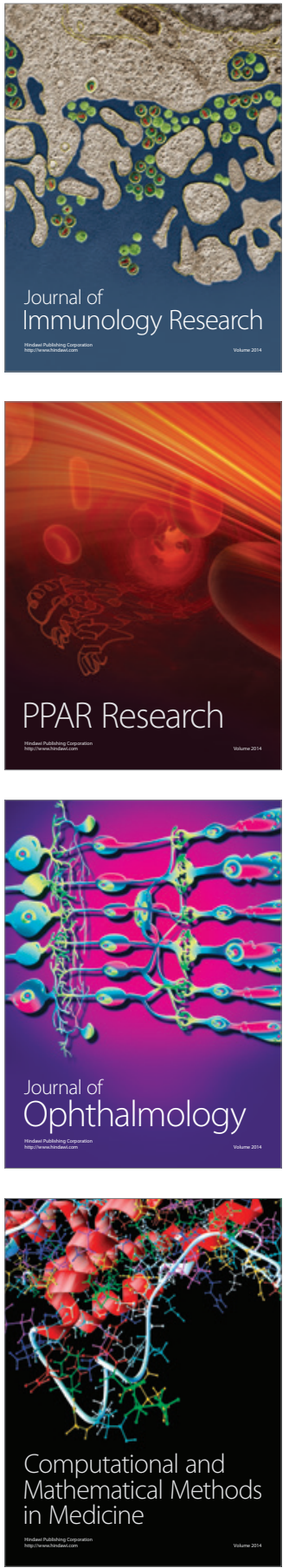

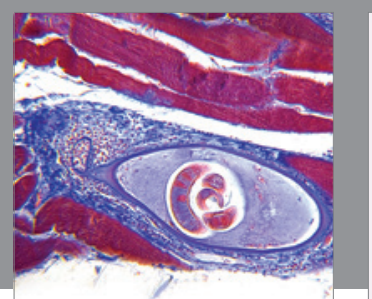

Gastroenterology Research and Practice

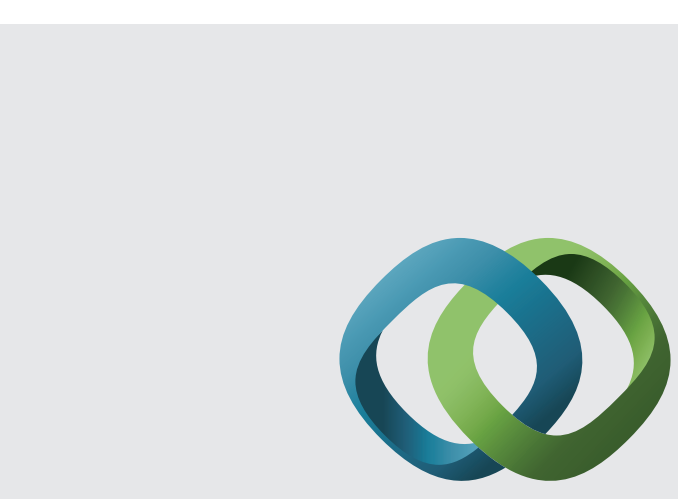

\section{Hindawi}

Submit your manuscripts at

http://www.hindawi.com
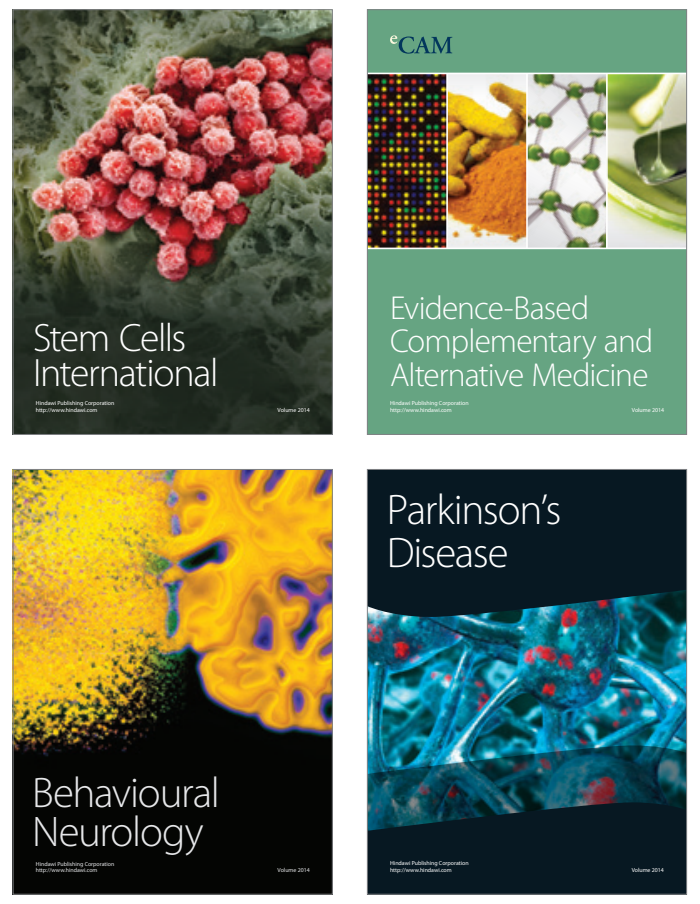
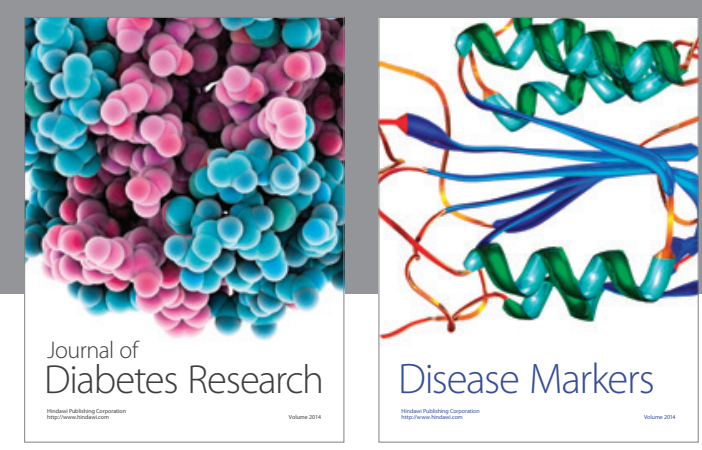

Disease Markers
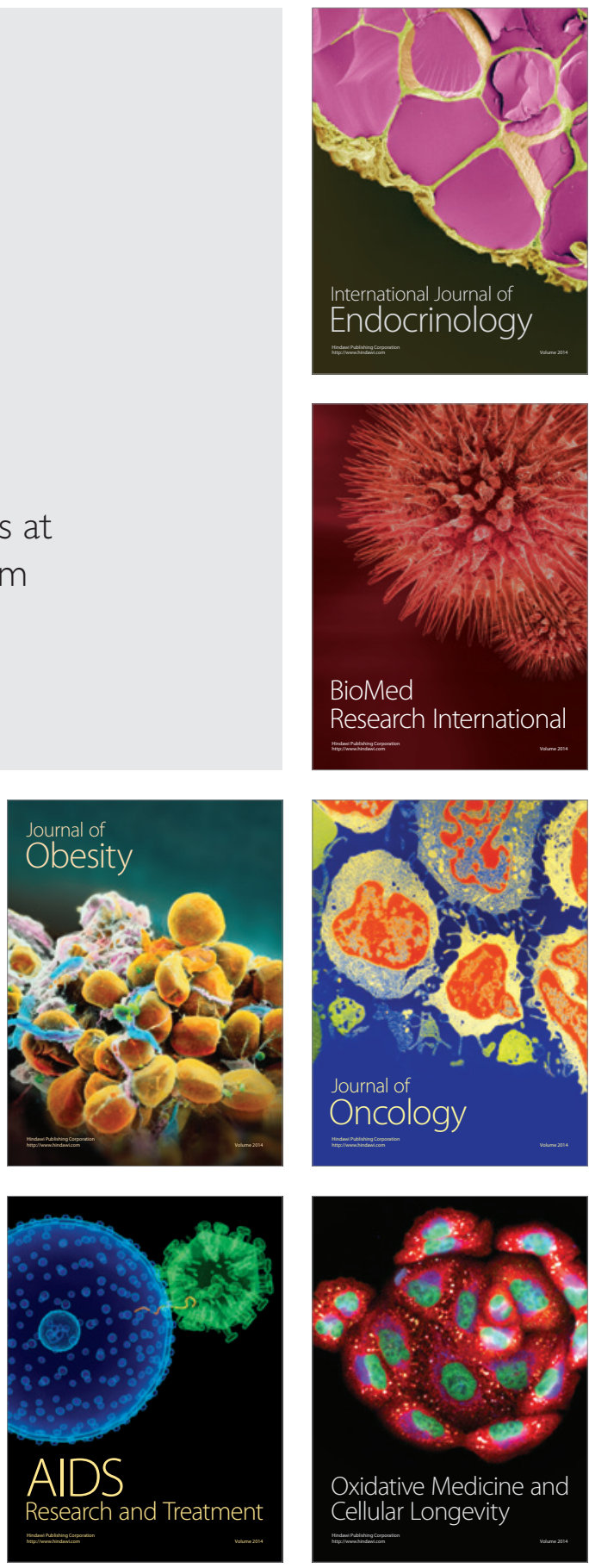